\title{
Genome-wide association study of pigmentary traits (skin and iris color) in individuals of East Asian ancestry
}

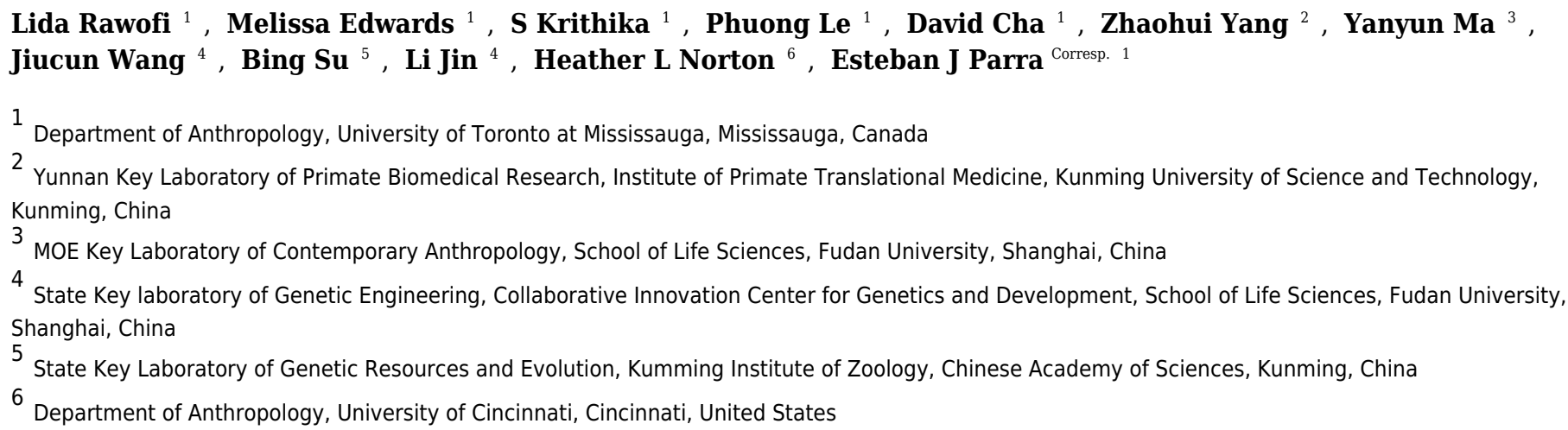

Background. Currently, there is limited knowledge about the genetics underlying pigmentary traits in East Asian populations. Here, we report the results of the first genome-wide association study of pigmentary traits (skin and iris color) in individuals of East Asian ancestry.

Methods. We obtained quantitative skin pigmentation measures (M-index) in the inner upper arm of the participants using a portable reflectometer $(\mathrm{N}=305)$. Quantitative measures of iris color (expressed as $L^{*}, a^{*}$ and $b^{*}$ CIELab coordinates) were extracted from high-resolution iris pictures $(\mathrm{N}=342)$. We also measured the color differences between the pupillary and ciliary regions of the iris (e.g. iris heterochromia). DNA samples were genotyped with Illumina's Infinium Multi-Ethnic Global Array (MEGA) and imputed using the 1000 Genomes Phase 3 samples as reference haplotypes.

Results. For skin pigmentation, we did not observe any genome-wide significant signal. We followed-up in three independent Chinese samples the lead SNPs of five regions showing multiple common markers (minor allele frequency $\geq 5 \%$ ) with good imputation scores and suggestive evidence of association ( $p$-values $<10-5$ ). One of these markers, rs2373391, which is located in an intron of the ZNF804B gene on chromosome 7, was replicated in one of the Chinese samples $(p=0.003)$. For iris color, we observed genome-wide signals in the OCA2 region on chromosome 15 . This signal is driven by the non-synonymous rs 1800414 variant, which explains $11.9 \%, 10.4 \%$ and $6 \%$ of the variation observed in the $b^{*}$, $a^{*}$ and $L^{*}$ coordinates in our sample, respectively. However, the OCA2 region was not associated with iris heterochromia.

Discussion. Additional genome-wide association studies in East Asian samples will be 
necessary to further disentangle the genetic architecture of pigmentary traits in East Asian populations. 
1 Genome-wide association study of pigmentary traits (skin and iris color) in individuals of 2 East Asian ancestry

3

4

Lida Rawofi ${ }^{1}$, Melissa Edwards ${ }^{1}$, S. Krithika ${ }^{1}$. Phuong Le ${ }^{1}$, David Cha ${ }^{1}$, Zhaohui Yang ${ }^{2}$, Yanyun $\mathrm{Ma}^{3}$, Jiucun Wang ${ }^{4}$, Bing Su${ }^{5}$, Li Jin ${ }^{4}$, Heather L. Norton ${ }^{6}$, Esteban J. Parra ${ }^{1}$

${ }^{1}$ Department of Anthropology, University of Toronto at Mississauga, Mississauga, ON, Canada ${ }^{2}$ Yunnan Key Laboratory of Primate Biomedical Research, Institute of Primate Translational Medicine, Kunming University of Science and Technology, Kunming, China ${ }^{3}$ MOE Key Laboratory of Contemporary Anthropology, School of Life Sciences, Fudan University, Shanghai, China

${ }^{4}$ State Key Laboratory of Genetic Engineering, Collaborative Innovation Center for Genetics and Development, School of Life Sciences, Fudan University, Shanghai, China.

${ }^{5}$ State Key Laboratory of Genetic Resources and Evolution, Kunming Institute of Zoology, Chinese Academy of Sciences, Kunming, China

${ }^{6}$ Department of Anthropology, University of Cincinnati, Cincinnati, USA

\section{Abstract}

Background. Currently, there is limited knowledge about the genetics underlying pigmentary traits in East Asian populations. Here, we report the results of the first genome-wide association study of pigmentary traits (skin and iris color) in individuals of East Asian ancestry.

Methods. We obtained quantitative skin pigmentation measures (M-index) in the inner upper arm of the participants using a portable reflectometer $(\mathrm{N}=305)$. Quantitative measures of iris color (expressed as L*, $\mathrm{a}^{*}$ and $\mathrm{b}^{*}$ CIELab coordinates) were extracted from high-resolution iris pictures $(\mathrm{N}=342)$. We also measured the color differences between the pupillary and ciliary regions of the iris (e.g. iris heterochromia). DNA samples were genotyped with Illumina's Infinium Multi-Ethnic Global Array (MEGA) and imputed using the 1000 Genomes Phase 3 samples as reference haplotypes.

Results. For skin pigmentation, we did not observe any genome-wide significant signal. We followed-up in three independent Chinese samples the lead SNPs of five regions showing multiple common markers (minor allele frequency $\geq 5 \%$ ) with good imputation scores and suggestive evidence of association ( $\mathrm{p}$-values $<10^{-5}$ ). One of these markers, rs2373391, which is located in an intron of the ZNF804B gene on chromosome 7, was replicated in one of the Chinese samples $(\mathrm{p}=0.003)$. For iris color, we observed genome-wide signals in the $O C A 2$ region on chromosome 15 . This signal is driven by the non-synonymous rs 1800414 variant, which explains $11.9 \%, 10.4 \%$ and $6 \%$ of the variation observed in the $b^{*}, a^{*}$ and $L^{*}$ coordinates in our sample, respectively. However, the $O C A 2$ region was not associated with iris heterochromia. Discussion. Additional genome-wide association studies in East Asian samples will be necessary to further disentangle the genetic architecture of pigmentary traits in East Asian populations.

\section{Introduction.}

Human pigmentation diversity is primarily driven by the type, amount and distribution of melanin in the skin, hair and iris. Pigmentation is a polygenic trait, and the last decade has witnessed numerous efforts to elucidate the genetic architecture of pigmentation through 
association and functional studies (Lamason et al., 2005; Kayser et al., 2008; Visser, Kayser \& Palstra 2012; Lui et al., 2015). There have also been important advances in the development of methods based on reflectance and bioimaging technologies, which have made it possible to obtain quantitative measurements of skin, hair and iris pigmentation (Liu et al., 2010; Walsh et al., 2011; Edwards et al., 2012; Beleza et al., 2013; Andersen et al., 2013; Norton et al., 2015; Edwards et al., 2015; Wollstein et al., 2017). As a result of these efforts, dozens of genetic markers have been associated with pigmentary phenotypes. However, the overwhelming majority of studies have focused on European populations, and there are still substantial gaps in our understanding of the genetic basis of pigmentation in other population groups.

Nonetheless, available research has shown skin lightening in Europe and East Asia to have occurred independently through convergent evolution. In Europe, markers within the $H E R C 2$ gene (e.g. rs12913832), which is close to OCA2, are strongly associated with blue eyes, and have also been associated with light skin pigmentation in GWAS. (Kayser et al., 2008; Lui et al., 2015; Sturm et al., 2008; Visser et al., 2012). The haplotype defined by rs12913832 is primarily restricted to Europe. In East Asia however, two non-synonymous variants in the OCA2 gene; rs1800414 (His615Arg) and rs74653330 (Ala481Thr) have been associated with light skin and eye color (Edwards et al., 2010; Abe et al., 2013; Eaton et al., 2015; Edwards et al., 2015; Norton et al., 2015; Yang et al 2016). Both of these polymorphisms are predicted to have a deleterious effect on the protein (Eaton et al., 2015). Functional studies have recently confirmed the role of rs1800414 in East Asian pigmentation (Yang et al., 2016). The haplotypes defined by rs 1800414 and rs74653330 are restricted to East Asia but they have a very different geographic distribution. The SNP rs1800414 is very frequent across East Asia while rs74653330 is primarily restricted to the Altaic speaking populations from Northern East Asia and Mongolia (Murray et al., 2015). There is general agreement that these two non-synonymous mutations arose long after the split of European and East Asian populations (Chen et al., 2015; Murray et al., 2015; Yang et al., 2016). In addition to variants in the $O C A 2$ gene, a non-synonymous mutation at rs 885479 (Arg163Gln) in $M C 1 R$ has also been association with pigmentation in East Asia (Yamaguchi et al., 2012). Interestingly, the derived $163 \mathrm{Gln}$ allele is present in very high frequencies in East Asian populations $(>60 \%)$, but very low frequencies in European and African populations.

Most of the studies conducted in East Asian populations have been candidate gene studies. Here, we report the results of the first genome wide association study of pigmentary traits in an East Asian population sample. Skin pigmentation was measured with a portable reflectometer while eye color was measured quantitatively (CIELab color space) from highresolution pictures of the iris. The samples were genotyped with Illumina's MEGA array, and imputed with the $1 \mathrm{KG}$ Phase 3 reference samples. We followed up the main signals observed in our GWAS in two independent East Asian samples. This study provides important insights about the architecture of skin pigmentation in East Asian populations.

\section{Materials and methods.}

Sample collection. 
87 Recruitment of study participants was carried out at the University of Toronto (Canada). All participants ranged between 18 and $35 \mathrm{yr}$ of age and were recruited using online and print advertisements directed towards the University of Toronto student community. A personal questionnaire was administered to each participant to determine their age, sex and whether or not they had been diagnosed with any pigmentation-related diseases or disorders.

Biogeographical ancestry was determined using information from the personal questionnaire, which inquired about the ancestry, place of birth and first language of each participant's maternal and paternal grandparents. The sample used in this study comprised individuals who had grandparents from China, Japan, South Korea or Taiwan. In the few cases where information about grandparents was not available, we used information about both parents to assess biogeographical ancestry. The total number of individuals included in the study was 425 . The study was approved by the University of Toronto Research and Ethics Board (Protocol Reference \#27015), and all participants provided written informed consent. A 2-ml saliva sample was obtained from each participant using the Oragene+DNA (OG-500) collection kit (DNA Genotek, Canada). All participants were instructed not to eat, drink or smoke for at least $30 \mathrm{~min}$ prior to obtaining the sample to ensure maximal sample purity. DNA was isolated from each sample using the protocol provided by DNA Genotek and eluted in $500 \mathrm{ml}$ of TE (10 mM Tris$\mathrm{HCl}, 1 \mathrm{mM}$ EDTA, pH 8.0) Buffer. Prior to genotyping, 23 DNA samples were excluded due to poor DNA quality. The final number of samples genotyped was 402 .

\section{Measurement of pigmentary traits.}

Skin pigmentation was measured quantitatively using the DSMII Dermaspectrometer (Cortex Technologies, Hadsund, Denmark), and melanin levels were reported as M index (individuals with higher M-values have darker pigmentation). Measurements were taken three times on the inner skin of the upper right arm and pigmentation was reported as the mean of the three measures, after excluding outliers. High-resolution pictures of the right iris of each participant were taken with a Fujifilm Finepix S3 Pro 12-megapixel DSLR mounted on a Nikor 105-mm macro lens. To control for lighting and exposure, photographs were taken with a coaxial biometric illuminator to deliver a constant and uniform source of light to each iris at 5,500 K (D55 illuminant). All photographs were taken under the same setting Delaneau et al., 2012) conditions, with an aperture of $\mathrm{f} / 19$, exposure sensitivity (ISO) set at 200 and a shutter speed of $1 / 125 \mathrm{~s}$. Iris pigmentation was digitally scored using a custom program designed to crop out both the pupil and sclera to retain only the iris. A wedge of the iris was then extracted, and color scores in CIELab coordinates were calculated from the pupillary and ciliary zones. In addition to the $\mathrm{L}^{*}, \mathrm{a}^{*}$ and $\mathrm{b}^{*}$ coordinates for the iris wedge, the program calculated the parameter delta, which describes color differences in the pupillary and ciliary regions of the iris. Detailed information about this program has been described in Edwards et al., (2015).

\section{Genotyping, phasing and imputation}

Genotyping was carried out with Illumina's Infinium Multi-Ethnic Global Array (MEGA) at the Clinical Genomics Centre (Mount Sinai Hospital, Toronto, Ontario, Canada) using standard protocols. The MEGA array, which includes approximately 1.7 million markers, was designed to 
127

128

129

130

131

132

133

134

135

136

137

138

139

140

141

142

143

144

145

146

147

148

149

150

151

152

153

154

155

156

157

158

159

160

161

162

163

164

165

166

capture common genome variation in diverse population groups. Four samples were included as blind duplicates, and the concordance rate was in all samples higher than $99.99 \%$. We used the program GenomeStudio to carry out the basic QC steps recommended by Illumina. After this initial QC step, approximately 1.4 million were retained for further analyses. The number of autosomal markers included was approximately 1.36 million. We performed additional QC steps to remove samples and markers, according to the following criteria, Sample QC: 1/ removal of samples with missing call rates $<0.9,2 /$ removal of samples that were outliers in Principal Component Analysis (PCA) plots, 3/ removal of samples with sex discrepancies, 4/ removal of samples that were outliers for heterozygosity, and 5/ removal of related individuals (pi-hat $>$ 0.2 ). Marker QC: $1 /$ removal of markers with genotype call rate $<0.95,2$ / removal of markers with Hardy-Weinberg p-values $<10^{-6}, 3$ / removal of Insertion/Deletion (Indel) markers, 4/ removal of markers with allele frequencies $<0.01,5 /$ removal of markers not present in the 1000 Genomes reference panel, or that do not match on chromosome, position and alleles, 6/ removal of $\mathrm{A} / \mathrm{T}$ or $\mathrm{G} / \mathrm{C}$ SNPs with MAF $>40 \%$ in the 1000 Genomes East Asian reference samples, and $7 /$ removal of SNPs with allele frequency differences $>20 \%$ between the study sample and the 1000 Genomes East Asian reference sample. After these QC steps, we retained 377 samples and 520,076 markers.

After performing the QC steps described above, the samples were phased using the program SHAPEIT2 (and imputed at the Sanger Imputation Service, using the Positional BurrowsWheeler Transform (PBWT) algorithm (Durbin, 2014), and the samples of the 1000 Genomes as reference haplotypes.

\section{Population structure}

We used the program EIGENSOFT to perform PCA and evaluate population stratification after pruning markers in high LD and removing regions showing high LD or genomic complexity.

\section{Statistical analyses.}

As a first step of the statistical analyses, we carried out a linear regression with M-values as the dependent variable, and sex and the first four Principal Component Axis as independent variables and saved the standardized residuals. A similar process was carried out for the L*, a*, $\mathrm{b}^{*}$ and delta iris values, but in this case, due to deviations from normality, the unstandardized residuals were transformed using the rank-based inverse normal transformation. The M-value residuals and the $\mathrm{L}^{*}, \mathrm{a}^{*}, \mathrm{~b}^{*}$ and delta transformed residuals were used as input for the association tests with the program SNPTEST v2 (Marchini and Howie, 2010), using an additive model and the expected test (e.g. using genotype dosages) in order to control for genotype uncertainty. For the $\mathrm{L}^{*}, \mathrm{a}^{*}, \mathrm{~b}^{*}$ coordinates that define iris color, we also run a Bayesian Multiple Phenotype test implemented in the program SNPTEST (-mpheno option). This test evaluates the three coordinates jointly and provides a $\log { }^{10}$ Bayes Factor reporting the ratio of two probabilities: the probability of the data under an unconstrained model $\left(\mathrm{M}_{1}\right)$, and the probability of the data under a null model $\left(\mathrm{M}_{0}\right)$ in which there is no effect. For example, a $\log { }^{10}$ Bayes Factor of 3 indicates that the probability of the data under the model M1 is 1000-fold higher than the probability of the data under the null model with no genotype effects. 
167 Of the 377 samples that were retained after the post-genotyping QC step, some samples had

168 missing phenotype data. The final number of samples with valid skin pigmentation data was 305 , and the final number of samples with valid iris color data was 342 .

\section{Annotation of genome-wide significant and suggestive signals.}

The genome-wide significant $\left(\mathrm{p}<10^{-8}\right)$ and suggestive signals $\left(\mathrm{p}<10^{-5}\right)$ identified in the statistical analyses were annotated using the online SNP-Nexus tool (http://snp-nexus.org/), which provides extensive annotations, including potential effects of non-synonymous coding SNPs on protein function (e.g. SIFT and Polyphen), potential regulatory effects (e.g. conserved transcription factor binding sites, microRNAs, Enhancers and $\mathrm{CpG}$ islands), evidence of evolutionary conservation (e.g. PHAST and GERP++) and evidence of association with complex diseases and disorders (e.g. GAD and NHGRI Catalogue of Published Genome-Wide Association studies).

Replication in independent East Asian samples.

The markers showing the strongest evidence of association with skin pigmentation were followed up in three independent Chinese samples, for which skin pigmentation measures (Mindex or L* values) were also available. The first two samples were collected by the Laboratory of Contemporary Anthropology at Fudan University between 2013 and 2015. Constitutive pigmentation was measured with a DSMII colormeter (Cortex Technology, Hadsund, Denmark) and pigmentation levels were reported as M-index values (darker pigmentation corresponds to higher M-index values). Pigmentation was estimated as the mean of three measures, after excluding outliers. The first sample was a cohort from the Jinan Military Hospital ( $\mathrm{N}=559)$. All the participants were male, and the mean age of this sample was 21.13 years. Constitutive pigmentation was measured in the buttocks during the month of September. The second sample was a cohort from Taizhou ( $\mathrm{N}=568)$, comprising 404 females and 164 males, with an average age of 44.32 years. Constitutive pigmentation was measured in the upper inner arm in the month of April. Genotyping of the SNPs was carried out using SNaPshot. In both samples, the association of the relevant markers with M-index values was carried out using linear regression, including age, rs 1800414 and sex (only for the Taizhou cohort) as covariates. The third sample comprises 346 Han Chinese individuals (College students) for whom constitutive pigmentation was measured in the buttocks and the upper inner arm. The study took place in the month of September. In this case, pigmentation was estimated as the mean of three measurements and reported using $\mathrm{L}^{*}$ values (darker pigmentation corresponds to lower $\mathrm{L}^{*}$ values). Genotyping was done using Sanger sequencing. The association of the variants with pigmentation was tested with the program PLINK, using sex and age as covariates. Unfortunately, to our knowledge, there have been no studies in East Asia reporting quantitative iris color estimates using the CIELab color space, so it was not possible to follow-up the signals identified for iris color in our East Asian samples.

\section{Results}

Distribution of pigmentary traits in East Asian sample. 
206 Supplementary Figure 1 shows the distribution of skin pigmentation values, expressed as the M-

207

208

209

210

211

212

213

214

215

216

217

218

219

220

221

222

223

224

225

226

227

228

229

230

231

232

233

234

235

236

237

238

239

240

241

242

243

244

245

index. The average M-value was 37.83 ( $\mathrm{SD}=2.843)$. Supplementary Figures 2A, 2B and 2C show the scatterplots of $\mathrm{a}^{*}$ and $\mathrm{b}^{*}, \mathrm{~L}^{*}$ and $\mathrm{a}^{*}$ and $\mathrm{L}^{*}$ and $\mathrm{b}^{*}$ iris color coordinates, respectively. The CIELab plots show that there is a substantial amount of variation in the three iris color coordinates, emphasizing the importance to use quantitative estimates of iris color, instead of categorical classifications (which in the case of this sample, would be restricted to "brown" color). Finally, the distribution of the delta values (difference in color coordinates between the pupillary and ciliary regions of the iris) are depicted in Supplementary Figure 3.

\section{Population structure}

Supplementary Figure 4 shows the representation of the first two axes of a PCA analysis of the East Asian sample included in this study, as well as the East Asian samples of the 1000 Genomes project (CDX: Chinese Dai in Xishuangbanna, KHV: Kinh in Ho Chi Minh City, Vietnam, CHB: Han Chinese in Beijing, CHS: Han Chinese South and JPT: Japanese in Tokyo). Most of the East Asian individuals included in this study overlap with the two Han Chinese samples (CHB and CHS) and a few individuals with the Japanese samples. Interestingly, the East Asian individuals who reported ancestry from Korea in our sample form a discrete cluster that is located between the Han Chinese and the Japanese 1000 Genomes samples.

\section{Results of Genome-Wide Association Study}

Figure 1 shows a Manhattan plot reporting the association results for skin pigmentation. We did not observe any genome-wide significant signal $\left(\mathrm{p}<5 \times 10^{-8}\right)$. Supplementary Figure 5 shows the QQ plot corresponding to skin pigmentation. There was no evidence of genomic inflation (lambda: 1.00). Table 1 reports the list of the common markers (maf $>5 \%$ ) with the lowest pvalues identified in the GWA study. All the markers listed in the Table have good imputation info scores (info $>0.8$ ), and correspond to regions with multiple significant markers (Supplementary Excel File). The regional plots for these regions are depicted in Supplementary Figure 6. Figure 2 shows the Manhattan plots reporting the association results for iris color (L*, $a^{*}, b^{*}$ and delta). The QQ plots for these traits are depicted in Supplementary Figure 7. Again, there is no evidence of genomic inflation for any of the traits. We observed a genome-wide signal in the well-known $O C A 2$ region. In this region, the top signal was rs 76930569, which had p-values of $9.27 \times 10^{-11}$ and $5.30 \times 10^{-12}$ for the $a^{*}$ and $b^{*}$ color dimensions, respectively. The Bayesian multiple phenotype test implemented in SNPTEST, based on the $\mathrm{L}^{*}, \mathrm{a}^{*}$ and $\mathrm{b}^{*}$ dimensions, also provided strong support for the $O C A 2$ region, with a $\log ^{10}$ Bayes Factor of 7 . In addition to the $O C A 2$ region, we identified numerous regions showing multiple markers with suggestive associations $\left(\mathrm{p}<10^{-5}\right)$ and good imputation scores. The lead signals for these regions are listed on Table 2, for each of the traits analyzed $\left(\mathrm{L}^{*}, \mathrm{a}^{*}, \mathrm{~b}^{*}\right.$ and delta). In order to facilitate interpretation, we provide in the Table the P-values for all the traits. It can be seen that all the regions show nominal significance in more than one iris color dimension. Additional information about relevant markers within each region is provided in the Supplementary Excel File. The regional plots are depicted in Supplementary Figures 8, 9, 10 and 11, respectively.

Replication of suggestive skin pigmentation signals. 
246 The lead SNPs showing suggestive evidence of association $\left(\mathrm{p}<10^{-5}\right)$ were followed up in three

247

248

249

250

251

252

253

254

255

256

257

258

259

260

261

262

263

264

265

266

267

268

269

270

271

272

273

274

275

276

277

278

279

280

281

282

283

284

285 independent Chinese samples, for which skin pigmentation measures in the buttocks or inner arm were available (M-index or L* values). The results of the replication effort are shown in Table 3. One of the markers, rs2373391 located on the ZNF804B gene on chromosome 7, was replicated in the Physical Examination cohort. In this sample, the A allele was significantly associated with lighter skin pigmentation $(\mathrm{p}=0.003$, Bonferroni-corrected significance threshold $\mathrm{p}=0.01$ ). This marker also had a concordant effect on the College student sample (A allele associated with lighter skin pigmentation -higher $\mathrm{L}^{*}$ values), but the $\mathrm{p}$-values were not nominally significant. In the Taizhou cohort, in which melanin values were measured in the upper arm, rs2373391 was also not significant and the regression coefficient was close to 0 . None of the remaining markers was nominally significant in any of the three replication samples.

\section{Association results for loci identified in European populations.}

We explored in our East Asian sample the allele frequencies and effect sizes of loci identified in European populations. These results are depicted in Supplementary Table 1. The table includes two non-synonymous variants in the genes SLC24A5 (rs1426654) and SLC45A2 (rs16891982), which have very high frequencies in Europe and very strong effects on skin pigmentation in admixed groups (Lamason et al., 2005; Norton et al., 2007). The table also includes variants that reached genome-wide significance in the largest GWAS of skin pigmentation in populations of European ancestry, which included more than 17,000 samples (Liu et al. 2015). Five of the six variants are either absent or present at very low frequencies in the East Asian sample and have relatively low imputation scores (info $<0.8$ ). The only exception is the polymorphism rs 4268748 located near the $M C 1 R$ gene, in which the minor allele (allele $\mathrm{C}$ ) has a frequency of $27.4 \%$ and a good imputation score (info $=0.983$ ). None of the variants reached nominal significance in our East Asian sample, except the SLC24A5 rs1426654 non-synonymous variant, which is present at a very low frequency in the sample (frequency of the derived A allele 1.6\%).

\section{Discussion}

In this paper, we describe the results of a genome-wide association study of pigmentary traits (skin pigmentation and iris color) in East Asian populations. We were able to confirm the important role that the gene OCA2 plays in East Asian populations. In our iris color GWA, we observed a genome-wide significant signal in the $O C A 2$ region. The lead SNP in this region was rs76930569, and this marker showed particularly strong associations with the $\mathrm{a}^{*}$ and $\mathrm{b}^{*}$ color dimensions ( $\mathrm{p}$-values of $9.27 \times 10^{-11}$ and $5.30 \times 10^{-12}$, respectively). However, rs 76930569 was not associated with delta, which is a measure that captures iris heterochromia (e.g. the color differences between the pupillary and ciliary regions of the iris). This indicates that the genetic architecture of iris color is different from the genetic architecture of iris heterochromia. It is important to note that rs76930569 is in very strong linkage disequilibrium with the nonsynonymous SNP rs $1800414\left(\mathrm{R}^{2}=1\right.$ in $\mathrm{CHB}$, CHS and JPT $1 \mathrm{KG}$ samples). Not surprisingly, the p-values of rs 1800414 were very similar to those of rs 76930569 in our sample, and most probably this non-synonymous SNP is the causal polymorphism driving the association. A recent 
286

287

288

289

290

291

292

293

294

295

296

297

298

299

300

301

302

303

304

305

306

307

308

309

310

311

312

313

314

315

316

317

318

319

320

321

322

323

324

325

326

study (Yang et al., 2016) using cultured melanocytes, and transgenic and targeted gene modification analyses on zebrafish and mouse have shown that the rs $1800414 \mathrm{G}$ variant (Arg615) is functional and leads to skin lightening. In our skin pigmentation analysis, the rs $1800414 \mathrm{G}$ variant was also associated with lighter skin pigmentation, but did not reach genome-wide significance ( $b e t a=-0.81, \mathrm{p}=5.5 \times 10^{-4}$ ). The magnitude of the skin pigmentation effect observed in our study is quite similar to the effects described in previous reports in which the M-index was used to describe constitutive pigmentation (e.g. Edwards et al., 2010, beta=1.26 in a sample of individuals of East Asian ancestry living in Canada, and beta=--0.86 in a Han Chinese sample; Eaton et al. 2015, beta $=-0.91$ in a sample of individuals of East Asian ancestry living in Canada).

Aside from the eye color signal in the $O C A 2$ region, no other regions surpassed the genome-wide significance threshold for skin pigmentation or iris color. However, there were several regions harboring multiple common markers with good imputation scores and suggestive $\mathrm{p}$-values $\left(\mathrm{p}<10^{-}\right.$ 5, Table 1 and Table 2; Supplementary Excel File). For skin pigmentation, we followed up the lead SNPs for each region in three independent Chinese samples (Table 3). One of the SNPs, rs2373391, which is located in an intron of the gene $Z N F 804 B$, replicated in one of the Chinese samples (beta $=-1.132, \mathrm{p}=0.003$ ). The gene $Z N F 804 B$ encodes a zinc finger protein, but it has been poorly characterized. Variants within this gene have been nominally associated with a number of traits in previous GWA studies (Anorexia nervosa, Wang et al., 2011; Heschl's gyrus morphology, Cai et al., 2014; and IgG glycosylation, Lauc et al., 2013, among others), but to our knowledge, no associations with pigmentary traits have been reported for this or nearby genes. Given that we were able to replicate the association only in one of the three East Asian samples, it will be important to investigate the potential role of this region in skin pigmentation in larger samples from East Asia. None of the other regions showing suggestive associations with skin pigmentation were replicated in the follow-up samples.

Unfortunately, we could not follow up any of the suggestive regions identified for iris color, because of the lack of studies in East Asia reporting quantitative measures of iris color. In this study, we show that there is a substantial amount of variation in iris color in East Asia (Supplementary Figure 2), and that the OCA2 region (and more particularly, the nonsynonymous variant rs 1800414) is a major determinant of the variation observed. We estimated that rs 1800414 explains $11.9 \%, 10.4 \%$ and $6 \%$ of the variation observed in the $b^{*}, a^{*}$ and $L^{*}$ coordinates in our sample, respectively. Therefore, there is a substantial amount of variation that remains unexplained in our sample. This highlights the need to carry out more studies using quantitative measures of iris color in East Asia. Categorical definitions of iris color would not be useful in East Asian populations, because in such a classification most of the irises would have been categorized as "brown", but there is a substantial range of $\mathrm{L}^{*}, \mathrm{a}^{*}$ and $\mathrm{b}^{*}$ values within this category.

To our knowledge, this is the first genome-wide association study of pigmentary traits in East Asian populations. The main weakness of this study is the small sample size, which limits our statistical power to identify variants with small effects on skin and iris pigmentation. Another limitation is that constitutive pigmentation was measured in different body sites (e.g. buttocks or 
327

328

329

330

331

332

333

334

335

336

337

338

339

340

341

342

343

344

345

346

347

348

349

350

351

352

353

354

355

356

357

358

359

360

361

362

363

364

365

366

367

368

inner arm) in the discovery and replication samples. Ideally, constitutive pigmentation should be measured in the buttocks, because there is less exposure to UV in this body site. However, often it is not possible to obtain pigmentation measures in the buttocks, and constitutive pigmentation is measured in the inner upper arm. In this site, there may be some exposure to UV light, particularly at certain times of the year, and several studies have reported that measurements of pigmentation in the inner arm are not interchangeable with estimates in the buttocks (Johansen et al. 2016; Bieliauskiene et al. 2017). In the discovery sample from Canada, inner upper arm measures were collected either in the winter/early spring (January to April) or the fall (October and November), so it would not be expected that UVR exposure would have a substantial effect on melanin levels. Similarly, in the two replication samples in which pigmentation measures were taken in the inner arm, Taizhou cohort and College students, the measures were collected in April, and September, respectively. Additionally, it is important to note that in one of the replication samples, pigmentation was reported as the CIELAB L* value, instead of the M-index. Although these two measures are highly correlated (Shriver and Parra, 2000), ideally all the discovery and replication samples should have been measured using the same index. Given that measurements were taken in different body sites, and for one replication sample using different pigmentation units, we could not carry out a meta-analysis of the discovery and replication samples. Instead, in our replication effort, we evaluated if the SNPs that were followed up in the replication samples reached significance after Bonferroni-correction based on the number of independent tests. Finally, it is important to note that for this GWAS we used both directly genotyped and imputed data. This is the standard protocol used in this type of studies, because it increases the power to identify associated variants (Spencer et al., 2009; Marchini and Howie, 2010). We implemented two different strategies to minimize potential problems related to imputation: 1) We used tests implemented in the program SNPTEST that take into account uncertainty in the imputed genotypes, and 2) We only followed up markers with very high imputation scores (info $>0.8$ ).

In spite of these limitations, this study provides important insights about the genetic architecture of skin and iris color in East Asian populations. Using the program Quanto (http://biostats.usc.edu/Quanto.html) we estimated that our skin pigmentation association study $(\mathrm{N}=305)$ had good power $(>0.8)$ to identify variants explaining approximately $12.5 \%$ of the variance of the trait. This implies that there are no polymorphic variants in our samples with large effects on skin melanin levels. For iris color $(\mathrm{N}=342)$, our study had good power to identify variants explaining approximately $11 \%$ of the variance of the trait, and in fact we identified a SNP with an effect of approximately that size. It will be critical to expand the number of genome-wide association studies in East Asian populations in order to be able to identify genetic markers with smaller effects on skin and iris pigmentation. We cannot exclude the possibility that there are variants with strong effects on pigmentation that have been fixed in East Asian populations due to the action of positive selection. This is something that happened in Europe with the well-known SLC24A5 rs1426654 A allele, which is the variant with the largest effect on melanin levels reported in human populations (Lamason et al., 2005). Identifying this type of variants, if they exist in East Asian populations, would require association studies in admixed samples with a substantial East Asian contribution. 
369 We followed up in our East Asian sample genetic markers in 6 loci that have been reported to

370

371

372

373

374

375

376

377

378

379

380

381

382

383

384

385

386

387

388

389

390

391

392

393

394

395

396

397

398

399

400

401

402

403

404

405

406

407

408

409

410

411 influence skin pigmentation in European populations (SLC45A5, IRF4, HERC2, SLC24A5, $D E F 8 / M C 1 R$ and $R A L Y / A S I P$, Supplementary Table 1). Most of these variants are absent (RALY/ASIP rs6059655) or present at very low frequencies in the East Asian sample (SLC45A2 rs 16891982, IRF4 rs12203592, HERC2 rs12913832 and SLC24A5 rs4268748, Supplementary Table 1). Because of their low frequencies, these SNPs explain little variation of skin pigmentation in East Asian populations. However, it is important to note that the estimated effect size of the derived SLC24A5 rs1426654 A allele in our GWAS is quite large (beta=-2.46), in agreement with numerous reports indicating that this is the locus with the strongest effect on skin pigmentation so far described in human populations (Lamason et al. 2005; Basu Mallick et al. 2013; Beleza et al., 2013). Only one of the variants reaching genome-wide significance in a large GWAS study in a European sample (DEF8/MC1R rs4268748; Liu et al., 2015) is present with relatively high frequencies in our East Asian sample. In fact, the allele reported to decrease pigmentation in the European sample (C allele) is slightly more frequent in East Asian populations than in European populations (28.5\% in EAS 1000 Genomes Project sample vs. $22.8 \%$ in EUR 1000 Genomes project). However, the rs 4268748 polymorphism is not nominally significant in our sample. One potential explanation is that rs4268748 is in linkage disequilibrium (LD) with causal loci in Europe, and the pattern of LD between rs4268748 and the causal loci, or the allele frequencies of the causal loci are different in European and East Asian populations. Supporting this explanation is the detailed analysis of the $M C 1 R$ region reported for one of the European samples (Rotterdam study) included in Liu et al. (2015) GWAS. These authors described that 3 known high-penetrance variants located within the MC1R gene (rs1805007 (R151C), rs1805008 (R160W), rs1805009 (D294H)) showed the most significant association with skin pigmentation in the Rotterdam study sample, and replacing the SNP rs4268748 by the high penetrance variants marginally increased the amount of the phenotypic variance explained. These three $M C 1 R$ high-penetrance variants, two of which have frequencies higher than 5\% in Europe (rs1805007 and rs1805008), are not found or are found in extremely low frequencies in East Asia, and this may explain the absence of an association signal in our study. Yamaguchi et al. (2012) reported that a low-penetrance MC1R variant, rs 885479 (R163Q), which is common in East Asia (frequency derived 163Q allele in EAS 1000 Genomes project sample $=61.6 \%$ ) but much less frequent in Europe was nominally associated with light skin in a Japanese sample. As expected, the rs 885479 polymorphism was present in our East Asian sample, with a frequency of the derived 163Q allele of $60.1 \%$. However, this variant was not nominally associated with skin pigmentation in our sample (beta $=-0.046, \mathrm{p}=0.843$ ).

Overall, our study confirm previous research indicating that the evolution of light skin in East Asia and Europe took place, at least to a large extent, independently in both groups after the split of the ancestral East Asian and European populations following the Out-of-Africa migration. In Europe, functional derived variants in the genes SLC24A5, SLC45A2 and OCA2/HERC2 increased in frequency as a result of positive selection (Lamason et al., 2005; Izaguirre et al., 2006; Norton et al., 2007; Lao et al., 2007; Wilde et al., 2014). In East Asia, the nonsynonymous polymorphism rs 1800414 located within the $O C A 2$ gene dramatically increased in frequency, also as a result of an independent selective event (Lao et al. 2007; Edwards et al., 2010; Donnelly et al., 2012; Hider et al., 2013). This constitutes, with lactase persistence and

Peer] reviewing PDF | (2017:08:20009:1:0:REVIEW 29 Sep 2017) 
412

413

414

415

416

417

418

419

420

421

422

423

424

425

426

427

428

429

430

431

432

433

434

435

436

437

438

439

440

441

442

443

444

445

446

447

448 adaptation to altitude, one of the most fascinating examples of convergent evolution in human populations.

\section{Conclusion}

Here we report the first genome-wide association of pigmentary traits (skin pigmentation and iris color) in East Asian populations. We measured these traits using quantitative methods, and we show that there is considerable variation not only in skin pigmentation but also in iris color, in spite of the fact that using categorical classifications, all the irises in this sample would have been categorized as "brown". We confirmed the important role that the OCA2 gene plays in normal pigmentation variation in East Asian populations. In particular, the non-synonymous variant rs 1800414 explains a substantial amount of variation in iris color. We did not observe any genome-wide significant variant for skin pigmentation, but one of the lead SNPs showing suggestive significance in our study, rs2373391, which is located in an intron of the ZNF804B gene on chromosome 7, was replicated in an independent Chinese sample $(p=0.003)$. It will be critical to carry out additional association studies in East Asian populations in order to uncover additional variants with smaller effects on skin pigmentation and iris color.

\section{Acknowledgments}

We would like to thank all the individuals that participated in this study.

\section{References}

Abe Y, Tamiya G, Nakamura T, Hozumi Y, and Suzuki T. 2013. Association of melanogenesis genes with skin color variation among Japanese females. Journal of Dermatological Science 69:167172. 10.1016/j.jdermsci.2012.10.016

Andersen JD, Johansen P, Harder S, Christoffersen SR, Delgado MC, Henriksen ST, Nielsen MM, Sørensen E, Ullum H, Hansen T, Dahl AL, Paulsen RR, Børsting C, Morling N. 2013. Genetic analyses of the human eye colours using a novel objective method for eye colour classification. Forensic Science International 7:508-515. 10.1016/j.fsigen.2013.05.003

Basu Mallick C, Iliescu FM, Möls M, Hill S, Tamang R, Chaubey G, Goto R, Ho SY, Gallego Romero I, Crivellaro F, Hudjashov G, Rai N, Metspalu M, Mascie-Taylor CG, Pitchappan R, Singh L, Mirazon-Lahr M, Thangaraj K, Villems R, Kivisild T. 2013. The light skin allele of SLC24A5 in South Asians and Europeans shares identity by descent. PLoS Genetics 9:e1003912. 10.1371/journal.pgen.1003912

Beleza S, Santos AM, McEvoy B, Alves I, Martinho C, Cameron E, Shriver MD, Parra EJ, and Rocha J. 2013. The Timing of Pigmentation Lightening in Europeans. Molecular Biology and Evolution 30:24-35. 10.1093/molbev/mss207 
Beleza S, Johnson NA, Candille SI, Absher DM, Coram MA, Lopes J, Campos J, Araújo II, Anderson TM, Vilhjálmsson BJ, Nordborg M, Correia E Silva A, Shriver MD, Rocha J, Barsh GS, Tang H. 2013. Genetic architecture of skin and eye color in an African-European admixed population. PLoS Genetics 9:e1003372. 10.1371/journal.pgen.1003372

Bieliauskiene G, Philipsen PA, Wulf HC. 2017. Can constitutive pigmentation be measured on upper inner arm? Correlation between arm and buttocks pigmentation. Photodermatology, Photoimmunology and Photomedicine 33:233-236. 10.1111/phpp.12314

Cai DC, Fonteijn H, Guadalupe T, Zwiers M, Wittfeld K, Teumer A, Hoogman M, Arias-Vasquez A, Yang Y, Buitelaar J, Fernandez G, Brunner HG, van Bokhoven H, Franke B, Hegenscheid K, Homuth G, Fisher SE, Grabe HJ, Francks C, and Hagoort P. 2014. A genome-wide search for quantitative trait loci affecting the cortical surface area and thickness of Heschl's gyrus. Genes Brain and Behavior 13:675-685. 10.1111/gbb.12157

Chen H, Hey J, and Slatkin M. 2015. A hidden Markov model for investigating recent positive selection through haplotype structure. Theoretical Population Biology 99:18-30. 10.1016/j.tpb.2014.11.001

Delaneau O, Marchini J, and Zagury JF. 2012. A linear complexity phasing method for thousands of genomes. Nature Methods 9:179-181. 10.1038/nmeth.1785

Donnelly MP, Paschou P, Grigorenko E, Gurwitz D, Barta C, Lu RB, Zhukova OV, Kim JJ, Siniscalco M, New M, Li H, Kajuna SL, Manolopoulos VG, Speed WC, Pakstis AJ, Kidd JR, Kidd KK. 2012. A global view of the OCA2-HERC2 region and pigmentation. Human Genetics 131:683696. 10.1007/s00439-011-1110-x

Durbin R. 2014. Efficient haplotype matching and storage using the positional Burrows-Wheeler transform (PBWT). Bioinformatics 30:1266-1272. 10.1093/bioinformatics/btu014

Eaton K, Edwards M, Krithika S, Cook G, Norton H, and Parra EJ. 2015. Association study confirms the role of two OCA2 polymorphisms in normal skin pigmentation variation in East Asian populations. American Journal of Human Biology 27:520-525. 10.1002/ajhb.22678

Edwards M, Bigham A, Tan JZ, Li SL, Gozdzik A, Ross K, Jin L, and Parra EJ. 2010. Association of the OCA2 Polymorphism His615Arg with Melanin Content in East Asian Populations: Further Evidence of Convergent Evolution of Skin Pigmentation. Plos Genetics 6. 10.1371/journal.pgen.1000867

Edwards M, Cha D, Krithika S, Johnson M, Cook G, and Parra EJ. 2016. Iris pigmentation as a quantitative trait: variation in populations of European, East Asian and South Asian ancestry and association with candidate gene polymorphisms. Pigment Cell \& Melanoma Research 29:141162. $10.1111 / \mathrm{pcmr} .12435$

Edwards M, Gozdzik A, Ross K, Miles J, and Parra EJ. 2012. Technical note: Quantitative measures of iris color using high resolution photographs. American Journal of Physical Anthropology 147:141-149. 10.1002/ajpa.21637

Hider JL, Gittelman RM, Shah T, Edwards M, Rosenbloom A, Akey JM, Parra EJ. 2013. Exploring signatures of positive selection in pigmentation candidate genes in populations of East Asian ancestry. BMC Evolutionary Biology 13:150. 10.1186/1471-2148-13-150 
Izagirre N, García I, Junquera C, de la Rúa C, Alonso S. 2006. A scan for signatures of positive selection in candidate loci for skin pigmentation in humans. Molecular Biology and Evolution 23:16971706.

Johansen P, Andersen JD, Madsen LN, Ullum H, Glud M, Børsting C, Gniadecki R, Morling N. 2016. Pigmentary Markers in Danes--Associations with Quantitative Skin Colour, Nevi Count, Familial Atypical Multiple-Mole, and Melanoma Syndrome. PLoS One 11:e0150381. 10.1371/journal.pone.0150381

Kayser M, Liu F, Janssens A, Rivadeneira F, Lao O, van Duijn K, Vermeulen M, Arp P, Jhamai MM, van Ijcken WFJ, den Dunnen JT, Heath S, Zelenika D, Despriet DDG, Klaver CCW, Vingerling JR, De Jong P, Hofman A, Aulchenko YS, Uitterlinden AG, Oostra BA, and van Duijn CM. 2008. Three genome-wide association studies and a linkage analysis identify HERC2 as a human iris color gene. American Journal of Human Genetics 82:411-423. 10.1016/j.ajhg.2007.10.003

Lamason RL, Mohideen M, Mest JR, Wong AC, Norton HL, Aros MC, Jurynec MJ, Mao XY, Humphreville VR, Humbert JE, Sinha S, Moore JL, Jagadeeswaran P, Zhao W, Ning G, Makalowska I, McKeigue PM, O'Donnell D, Kittles R, Parra EJ, Mangini NJ, Grunwald DJ, Shriver MD, Canfield VA, and Cheng KC. 2005. SLC24A5, a putative cation exchanger, affects pigmentation in zebrafish and humans. Science 310:1782-1786. 10.1126/science.1116238

Lao O, de Gruijter JM, van Duijn K, Navarro A, Kayser M. 2007. Signatures of positive selection in genes associated with human skin pigmentation as revealed from analyses of single nucleotide polymorphisms. Annals of Human Genetics 71:354-369.

Lauc G, Huffman JE, Pucic M, Zgaga L, Adamczyk B, Muzinic A, Novokmet M, Polasek O, Gornik O, Kristic J, Keser T, Vitart V, Scheijen B, Uh HW, Molokhia M, Patrick AL, McKeigue P, Kolcic I, Lukic IK, Swann O, van Leeuwen FN, Ruhaak LR, Houwing-Duistermaat JJ, Slagboom PE, Beekman M, de Craen AJM, Deelder AM, Zeng Q, Wang W, Hastie ND, Gyllensten U, Wilson JF, Wuhrer M, Wright AF, Rudd PM, Hayward C, Aulchenko Y, Campbell H, and Rudan I. 2013. Loci Associated with N-Glycosylation of Human Immunoglobulin G Show Pleiotropy with Autoimmune Diseases and Haematological Cancers. Plos Genetics 9. 10.1371/journal.pgen.1003225

Liu F, Visser M, Duffy DL, Hysi PG, Jacobs LC, Lao O, Zhong KY, Walsh S, Chaitanya L, Wollstein A, Zhu G, Montgomery GW, Henders AK, Mangino M, Glass D, Bataille V, Sturm RA, Rivadeneira F, Hofman A, van Ijcken WFJ, Uitterlinden AG, Palstra R, Spector TD, Martin NG, Nijsten TEC, and Kayser M. 2015. Genetics of skin color variation in Europeans: genome-wide association studies with functional follow-up. Human Genetics 134:823-835. 10.1007/s00439-015-1559-0

Liu F, Wollstein A, Hysi PG, Ankra-Badu GA, Spector TD, Park D, Zhu G, Larsson M, Duffy DL, Montgomery GW, Mackey DA, Walsh S, Lao O, Hofman A, Rivadeneira F, Vingerling JR, Uitterlinden AG, Martin NG, Hammond CJ, and Kayser M. 2010. Digital Quantification of Human Eye Color Highlights Genetic Association of Three New Loci. Plos Genetics 6. 10.1371/journal.pgen.1000934

Marchini J, and Howie B. 2010. Genotype imputation for genome-wide association studies. Nature Reviews Genetics 11:499-511. 10.1038/nrg2796

Murray N, Norton H, and Parra EJ. 2015. Distribution of two OCA2 polymorphisms associated with pigmentation in East Asian populations. Human Genome Variation 2:15058 
530

531

532

533

534

535

536

537

538

539

540

541

542

543

544

545

546

547

548

549

550

551

552

553

554

555

556

557

558

559

560

561

562

563

564

565

566

567

568

569

570

Norton HL1, Kittles RA, Parra E, McKeigue P, Mao X, Cheng K, Canfield VA, Bradley DG, McEvoy B, Shriver MD. 2007. Genetic evidence for the convergent evolution of light skin in Europeans and East Asians. Molecular Biology and Evolution 24:710-722.

Norton HL, Edwards M, Krithika S, Johnson M, Werren EA, and Parra EJ. 2016. Quantitative Assessment of Skin, Hair, and Iris Variation in a Diverse Sample of Individuals and Associated Genetic Variation. American Journal of Physical Anthropology 160:570-581. 10.1002/ajpa.22861

Shriver MD, Parra EJ. 2000. Comparison of narrow-band reflectance spectroscopy and tristimulus colorimetry for measurements of skin and hair color in persons of different biological ancestry. American Journal of Physical Anthropology 112:17-27.

Spencer CC, Su Z, Donnelly P, Marchini J. 2009. Designing genome-wide association studies: sample size, power, imputation, and the choice of genotyping chip. PLoS Genetics 5:e1000477. 10.1371/journal.pgen.1000477

Visser M, Kayser M, and Palstra RJ. 2012. HERC2 rs12913832 modulates human pigmentation by attenuating chromatin-loop formation between a long-range enhancer and the OCA2 promoter. Genome Research 22:446-455. 10.1101/gr.128652.111

Walsh S, Lindenbergh A, Zuniga SB, Sijen T, de Knijff P, Kayser M, and Ballantyne KN. 2011. Developmental validation of the IrisPlex system: Determination of blue and brown iris colour for forensic intelligence. Forensic Science International-Genetics 5:464-471. 10.1016/j.fsigen.2010.09.008

Wang K, Zhang H, Bloss CS, Duvvuri V, Kaye W, Schork NJ, Berrettini W, Hakonarson H, and Price Fdn Collaborative G. 2011. A genome-wide association study on common SNPs and rare CNVs in anorexia nervosa. Molecular Psychiatry 16:949-959. 10.1038/mp.2010.107

Wilde S, Timpson A, Kirsanow K, Kaiser E, Kayser M, Unterländer M, Hollfelder N, Potekhina ID, Schier W, Thomas MG, Burger J. 2014. Direct evidence for positive selection of skin, hair, and eye pigmentation in Europeans during the last 5,000 y. Proceedings of the National Academy of Sciences USA 111:4832-4837. 10.1073/pnas.1316513111

Wollstein A, Walsh S, Liu F, Chakravarthy U, Rahu M, Seland JH, Soubrane G, Tomazzoli L, Topouzis F, Vingerling JR, Vioque J, Bohringer S, Fletcher AE, and Kayser M. 2017. Novel quantitative pigmentation phenotyping enhances genetic association, epistasis, and prediction of human eye colour. Scientific Reports 7. 10.1038/srep43359

Yamaguchi K, Watanabe C, Kawaguchi A, Sato T, Naka I, Shindo M, Moromizato K, Aoki K, Ishida H, and Kimura R. 2012. Association of melanocortin 1 receptor gene (MC1R) polymorphisms with skin reflectance and freckles in Japanese. Journal of Human Genetics 57:700-708. 10.1038/jhg.2012.96

Yang ZH, Zhong H, Chen J, Zhang XM, Zhang H, Luo X, Xu SH, Chen H, Lu DS, Han YL, Li JK, Fu LJ, Qi XB, Peng Y, Xiang K, Lin Q, Guo Y, Li M, Cao XY, Zhang YF, Liao SY, Peng YM, Zhang L, Guo XS, Dong SS, Liang F, Wang J, Willden A, Aun HS, Serey B, Sovannary T, Bunnath L, Samnom H, Mardon G, Li QW, Meng AM, Shi H, and Su B. 2016. A Genetic Mechanism for Convergent Skin Lightening during Recent Human Evolution.Molecular Biology and Evolution 33:1177-1187. 10.1093/molbev/msw003 
574

575

576

577 


\section{Figure 1 (on next page)}

Manhattan plot reporting the association results for skin pigmentation. 


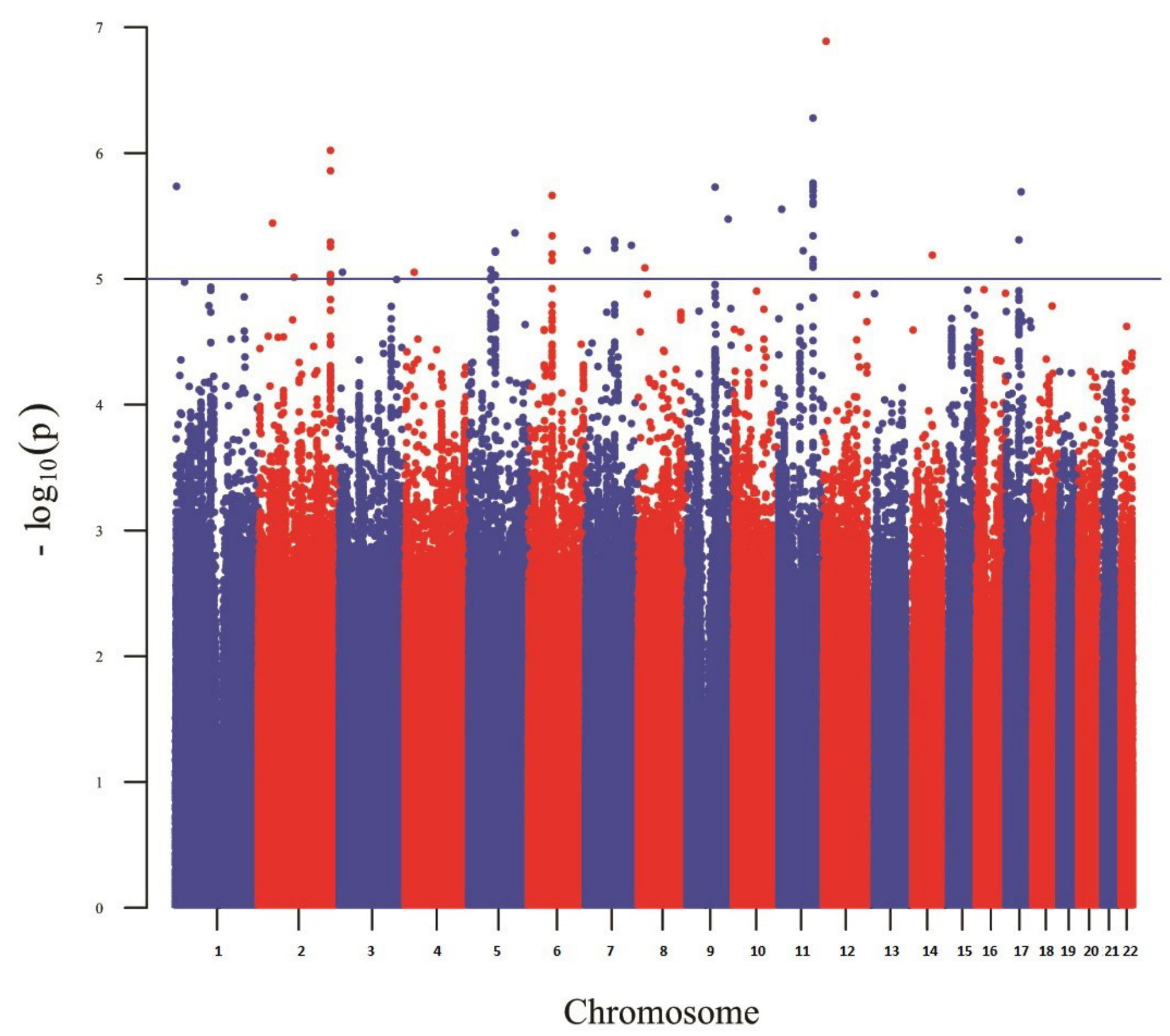


Figure 2 (on next page)

Manhattan plot reporting the association results for iris color ( $L^{*}, a^{*}, b^{*}$ and delta) 
PeerJ

$L^{*}$

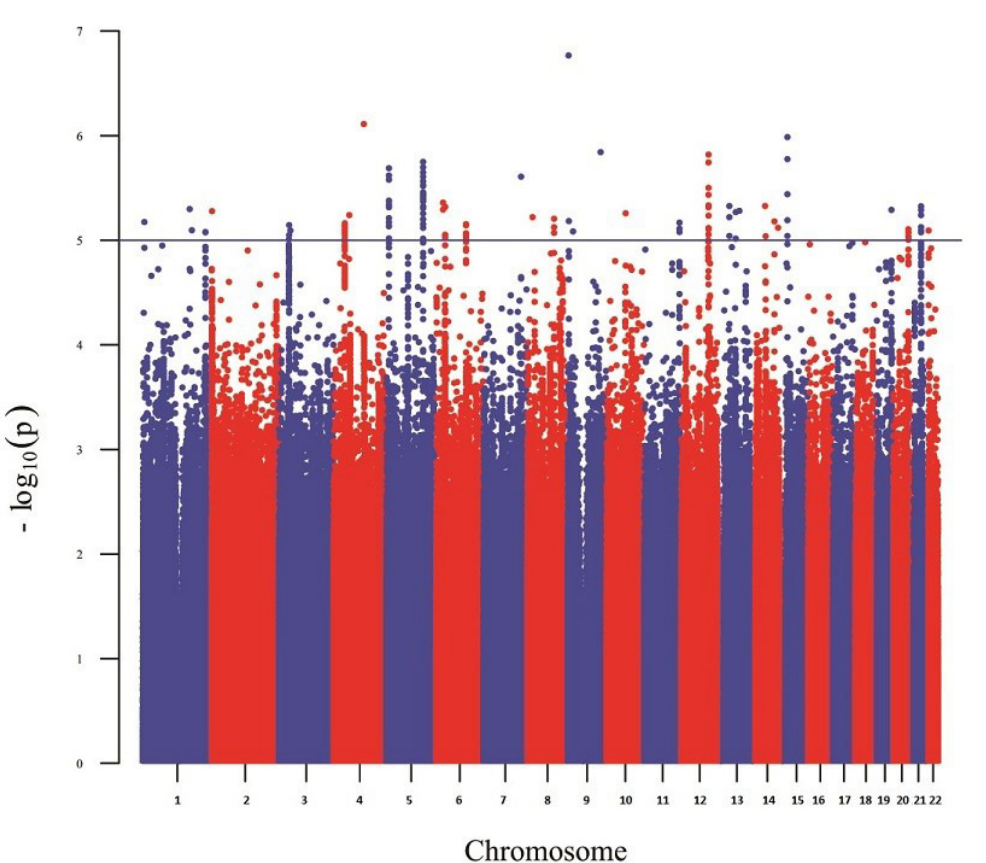

$b^{*}$

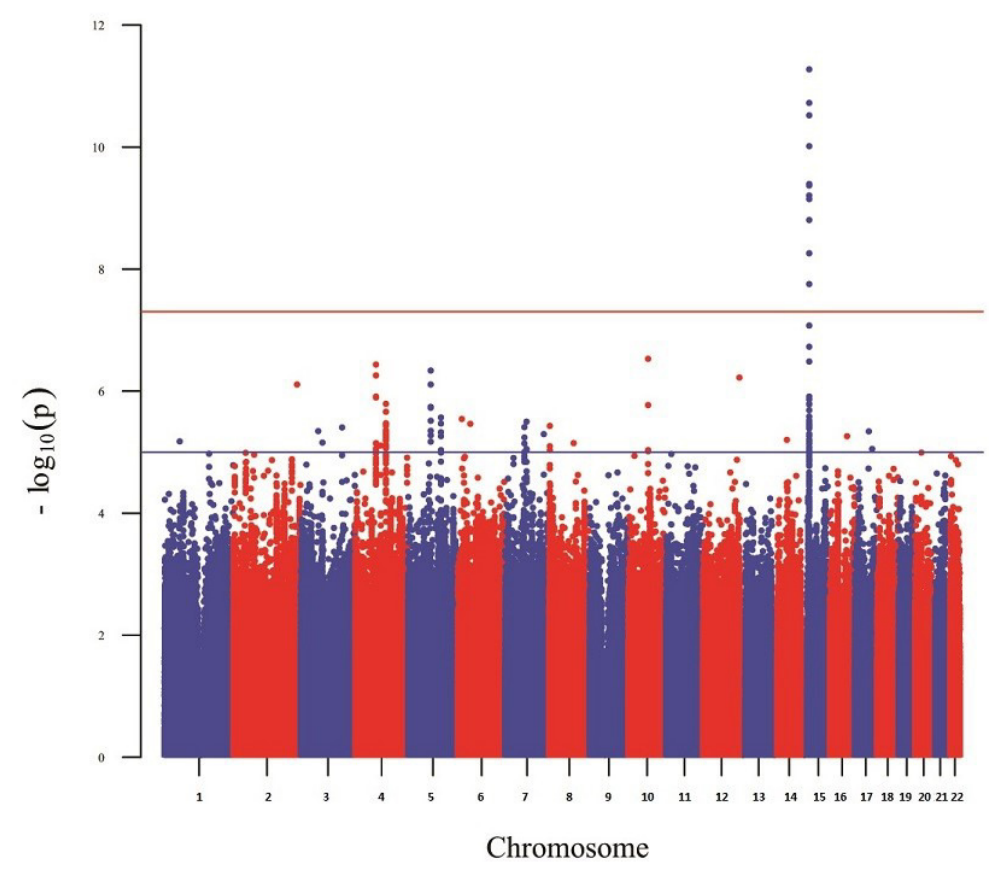

Manuscript to be reviewed

a*

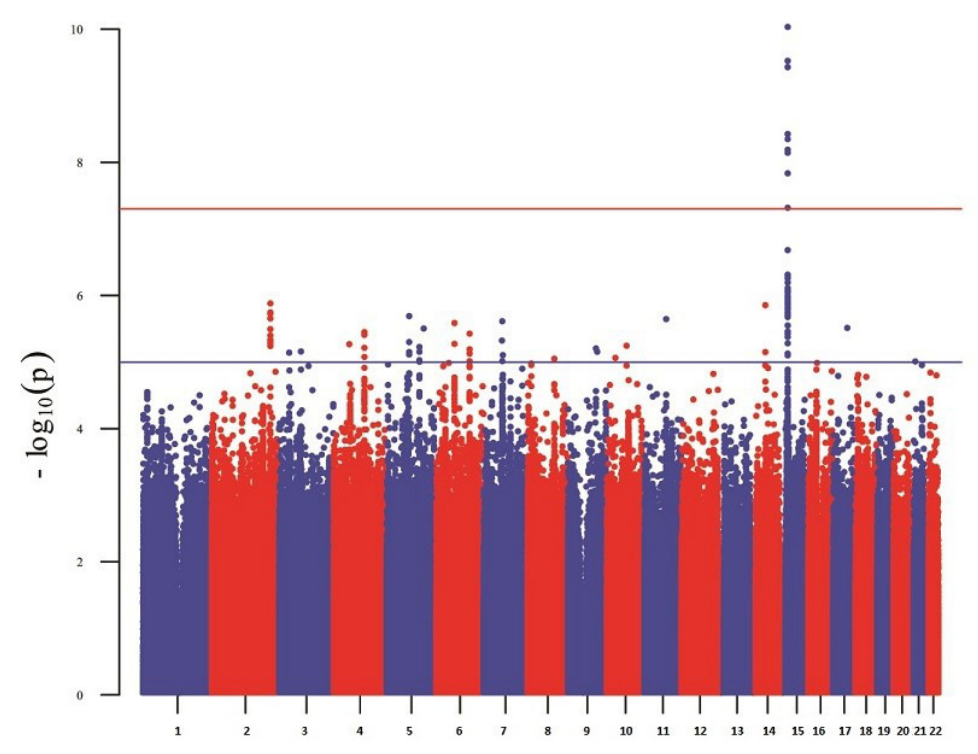

Chromosome

Delta

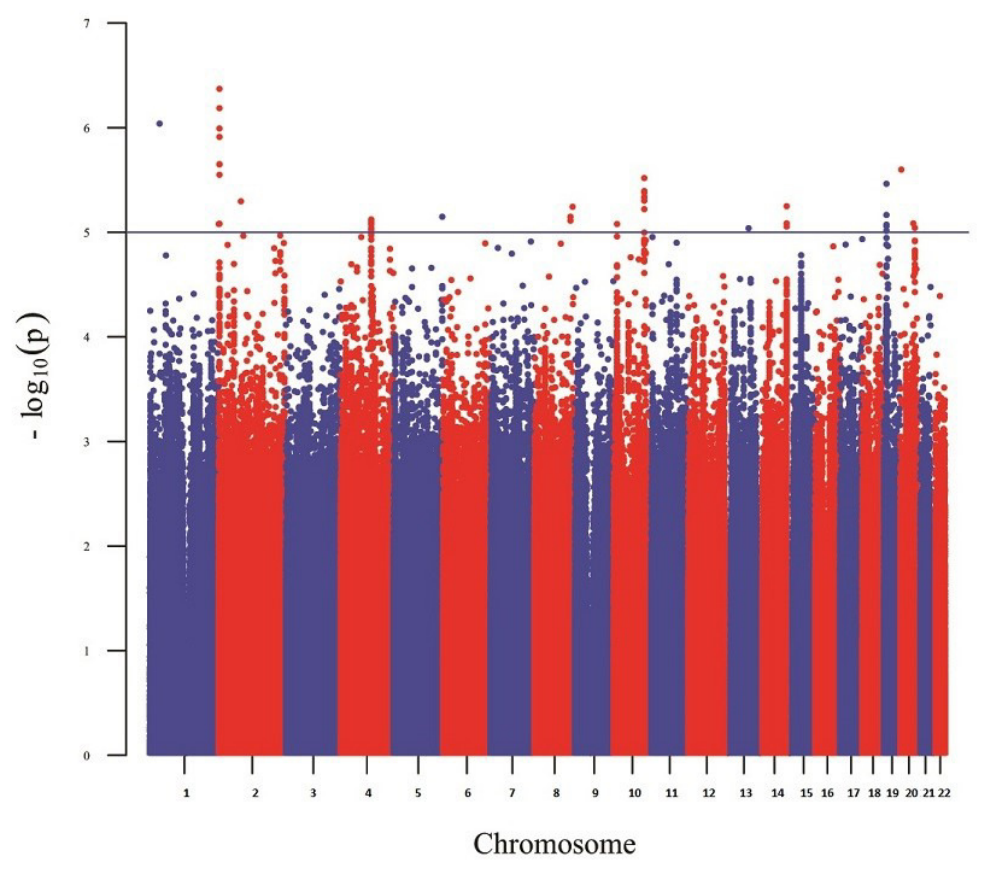

Peer) reviewing PDF | (2017:08:20009:1:0:REVIEW 29 Sep 2017) 


\section{Table $\mathbf{1}$ (on next page)}

Main signals observed in the GWAS of skin pigmentation (M-values). We report the beta, SE and P-values obtained with the model using the standardized and unstandardized residuals. 
1 Table 1. Main signals observed in the GWAS of skin pigmentation (M-values). We report the beta, SE and P-values obtained with the model using 2 the standardized and unstandardized residuals.

\begin{tabular}{|c|c|c|c|c|c|c|c|c|c|c|c|c|}
\hline SNP & $\mathrm{CHR}$ & POS & NEA/EA & Gene & Frq EA & INFO & Beta $^{1}$ & $\mathrm{SE}^{1}$ & $\mathrm{P}^{1}$ & Beta $^{2}$ & $\mathrm{SE}^{2}$ & $\mathrm{P}^{2}$ \\
\hline rs2003589 & 2 & 217527465 & $\mathrm{~T} / \mathrm{C}$ & IGFBP2 & 0.063 & 0.843 & 0.885 & 0.177 & $9.54 \mathrm{E}-07$ & 2.464 & 0.494 & $1.05 \mathrm{E}-06$ \\
\hline rs853807 & 5 & 67752638 & $\mathrm{C} / \mathrm{T}$ & & 0.485 & 0.956 & -0.347 & 0.077 & $8.45 E-06$ & -0.961 & 0.213 & $9.75 \mathrm{E}-06$ \\
\hline rs57836066 & 6 & 71304950 & $\mathrm{~T} / \mathrm{G}$ & $R P 11-134 K 13.4$ & 0.086 & 0.979 & -0.661 & 0.137 & 2.17E-06 & -1.838 & 0.382 & $2.40 \mathrm{E}-06$ \\
\hline rs2373391 & 7 & 88449300 & $\mathrm{~T} / \mathrm{A}$ & ZNF804B & 0.681 & 0.987 & -0.384 & 0.083 & $4.98 \mathrm{E}-06$ & -1.076 & 0.232 & 5.09E-06 \\
\hline rs7945369 & 11 & 103425586 & $\mathrm{~T} / \mathrm{C}$ & & 0.537 & 0.968 & -0.405 & 0.079 & $5.26 \mathrm{E}-07$ & -1.159 & 0.224 & 3.97E-07 \\
\hline
\end{tabular}

4

5

6

7

8

9

10

11

12

13

14

15

16

17

18

19

20

21

22

${ }^{1}$ Beta estimate using standardized residuals

${ }^{2}$ Beta estimate using unstandardized residuals 


\section{Table 2 (on next page)}

Main signals observed in the GWAS of iris color ( $L^{*}, a^{*}, b^{*}$ and delta).

For each trait, we report the P-values obtained for all the other traits in the association study, as well as the $\log 10$ Bayes Factor obtained in the joint analysis of $L^{*}, a^{*}$ and $b^{*}$. 
1 Table 2. Main signals observed in the GWAS of iris color $\left(L^{*}, a^{*}, b^{*}\right.$ and delta). For each trait, we report the P-values obtained for all the other 2 traits in the association study, as well as the $\log 10$ Bayes Factor obtained in the joint analysis of $L^{*}, a^{*}$ and $b^{*}$. 


\begin{tabular}{|c|c|c|c|c|c|c|c|c|c|c|c|c|c|}
\hline & $\mathrm{CHR}$ & POS & NEA/EA & Gene & Frq EA & INFO & Beta & SE & $\mathrm{P}\left(\mathrm{L}^{*}\right)$ & $\mathrm{P}(\mathrm{a} *)$ & $\mathrm{P}\left(\mathrm{b}^{*}\right)$ & $\mathrm{P}$ (delta) & $\mathrm{BF}$ \\
\hline \multicolumn{14}{|l|}{ L*signals } \\
\hline rs6664080 & 1 & 226797426 & $\mathrm{~T} / \mathrm{C}$ & C1orf95 & 0.297 & 0.993 & 0.362 & 0.08 & 8.36E-06 & $2.56 \mathrm{E}-03$ & $2.88 \mathrm{E}-02$ & $3.42 \mathrm{E}-03$ & 2.774 \\
\hline rs4425211 & 3 & 36951898 & $A / G$ & TRANK1 & 0.072 & 0.959 & -0.676 & 0.148 & $7.14 \mathrm{E}-06$ & $6.79 \mathrm{E}-05$ & $6.33 \mathrm{E}-04$ & $5.85 \mathrm{E}-01$ & 1.678 \\
\hline rs12501370 & 4 & 41043870 & $\mathrm{G} / \mathrm{C}$ & $A P B B 2$ & 0.556 & 0.982 & -0.332 & 0.073 & $6.89 \mathrm{E}-06$ & $3.14 \mathrm{E}-04$ & $1.93 \mathrm{E}-03$ & 7.97E-01 & 2.501 \\
\hline rs2658084 & 5 & 10136135 & $\mathrm{C} / \mathrm{T}$ & CTD-219904.1 & 0.835 & 0.97 & -0.502 & 0.104 & $2.05 \mathrm{E}-06$ & $4.11 \mathrm{E}-03$ & $3.32 \mathrm{E}-02$ & $2.29 \mathrm{E}-01$ & 2.976 \\
\hline rs113633047 & 5 & 133402895 & $\mathrm{G} / \mathrm{T}$ & & 0.282 & 0.95 & 0.419 & 0.087 & $2.00 \mathrm{E}-06$ & $2.25 \mathrm{E}-04$ & $5.94 \mathrm{E}-04$ & $1.09 \mathrm{E}-01$ & 2.735 \\
\hline rs6924266 & 6 & 25505617 & $\mathrm{C} / \mathrm{T}$ & LRRC16A & 0.233 & 0.98 & -0.408 & 0.087 & $4.38 \mathrm{E}-06$ & $3.64 \mathrm{E}-05$ & $1.05 \mathrm{E}-04$ & $8.77 \mathrm{E}-02$ & 2.583 \\
\hline NA & 6 & 32626040 & $\mathrm{C} / \mathrm{T}$ & & 0.075 & 0.996 & -0.64 & 0.138 & $4.81 \mathrm{E}-06$ & $6.29 \mathrm{E}-05$ & $1.79 \mathrm{E}-03$ & $2.07 \mathrm{E}-01$ & 2.003 \\
\hline rs9373973 & 6 & 108079595 & T/A & SCML4 & 0.185 & 0.938 & -0.454 & 0.099 & 7.02E-06 & 2.19E-03 & $1.12 \mathrm{E}-02$ & $9.11 \mathrm{E}-02$ & 2.36 \\
\hline rs145048184 & 8 & 97070341 & $\mathrm{C} / \mathrm{T}$ & & 0.084 & 0.962 & 0.643 & 0.14 & $6.23 \mathrm{E}-06$ & $8.89 \mathrm{E}-06$ & $3.05 \mathrm{E}-05$ & $5.74 \mathrm{E}-01$ & 1.947 \\
\hline rs610106 & 11 & 128935028 & $T / C$ & ARHGAP32 & 0.814 & 0.97 & 0.44 & 0.096 & $6.79 \mathrm{E}-06$ & $9.91 \mathrm{E}-04$ & $5.40 \mathrm{E}-03$ & 9.39E-01 & 2.318 \\
\hline rs249625 & 12 & 98185248 & $A / G$ & & 0.584 & 0.979 & -0.364 & 0.077 & $3.16 \mathrm{E}-06$ & $5.72 \mathrm{E}-03$ & $3.77 \mathrm{E}-03$ & $9.27 \mathrm{E}-03$ & 3.352 \\
\hline rs76930569 & 15 & 28196145 & $\mathrm{C} / \mathrm{T}$ & OCA2 & 0.623 & 1 & 0.38 & 0.076 & $1.03 \mathrm{E}-06$ & $9.27 \mathrm{E}-11$ & $5.30 \mathrm{E}-12$ & $3.42 \mathrm{E}-01$ & 7.007 \\
\hline rs75161997 & 20 & 55701691 & $\mathrm{C} / \mathrm{T}$ & & 0.06 & 0.992 & -0.732 & 0.161 & $7.84 \mathrm{E}-06$ & $7.49 \mathrm{E}-04$ & $1.77 \mathrm{E}-03$ & $8.34 \mathrm{E}-01$ & 1.485 \\
\hline rs8131065 & 21 & 38011676 & $\mathrm{C} / \mathrm{T}$ & & 0.215 & 0.979 & -0.419 & 0.09 & $4.75 \mathrm{E}-06$ & $1.49 \mathrm{E}-02$ & $1.25 \mathrm{E}-01$ & $4.43 \mathrm{E}-02$ & 3.478 \\
\hline \multicolumn{14}{|l|}{ a*signals } \\
\hline rs55821297 & 4 & 111399598 & $A / G$ & ENPEP & 0.771 & 0.926 & 0.435 & 0.092 & $2.96 \mathrm{E}-04$ & $3.55 \mathrm{E}-06$ & $2.19 \mathrm{E}-06$ & $4.86 \mathrm{E}-05$ & 2.710 \\
\hline rs72763726 & 5 & 82044846 & $\mathrm{C} / \mathrm{T}$ & & 0.090 & 0.950 & 0.652 & 0.135 & $1.32 \mathrm{E}-04$ & $2.04 \mathrm{E}-06$ & $4.60 \mathrm{E}-07$ & $7.57 \mathrm{E}-01$ & 2.349 \\
\hline rs330203 & 5 & 119016414 & $\mathrm{C} / \mathrm{G}$ & CTC-507E12.1 & 0.500 & 0.996 & -0.343 & 0.075 & $4.05 \mathrm{E}-03$ & $7.02 \mathrm{E}-06$ & $3.40 \mathrm{E}-06$ & $8.74 \mathrm{E}-01$ & 2.997 \\
\hline rs9345521 & 6 & 65511281 & $\mathrm{C} / \mathrm{A}$ & $E Y S$ & 0.467 & 0.976 & 0.352 & 0.074 & 1.64E-04 & $2.59 \mathrm{E}-06$ & $6.92 \mathrm{E}-05$ & $5.27 \mathrm{E}-01$ & 2.879 \\
\hline rs78001527 & 6 & 120168240 & $\mathrm{G} / \mathrm{C}$ & & 0.074 & 0.973 & -0.686 & 0.146 & $1.65 \mathrm{E}-03$ & $3.74 \mathrm{E}-06$ & $6.68 \mathrm{E}-05$ & $9.24 \mathrm{E}-01$ & 1.892 \\
\hline rs6977845 & 7 & 67216370 & $\mathrm{~T} / \mathrm{A}$ & & 0.093 & 0.881 & 0.666 & 0.139 & $2.58 \mathrm{E}-04$ & $2.42 \mathrm{E}-06$ & 5.04E-05 & $7.82 \mathrm{E}-01$ & 1.979 \\
\hline rs76930569 & 15 & 28196145 & $\mathrm{C} / \mathrm{T}$ & OCA2 & 0.623 & 1.000 & 0.497 & 0.074 & $1.03 \mathrm{E}-06$ & $9.27 \mathrm{E}-11$ & $5.30 \mathrm{E}-12$ & $3.42 \mathrm{E}-01$ & 7.007 \\
\hline \multicolumn{14}{|l|}{ b*signals } \\
\hline rs12510870 & 4 & 74358277 & $\mathrm{~T} / \mathrm{C}$ & $A F M$ & 0.190 & 0.994 & -0.496 & 0.096 & $1.06 \mathrm{E}-01$ & $3.37 \mathrm{E}-04$ & $3.68 \mathrm{E}-07$ & $1.60 \mathrm{E}-01$ & 4.614 \\
\hline rs1996603 & 4 & 111378362 & $\mathrm{G} / \mathrm{A}$ & ENPEP & 0.779 & 0.960 & 0.451 & 0.092 & $1.32 \mathrm{E}-03$ & $1.22 \mathrm{E}-05$ & $1.61 \mathrm{E}-06$ & $1.60 \mathrm{E}-03$ & 2.728 \\
\hline rs72763726 & 5 & 82044846 & $\mathrm{C} / \mathrm{T}$ & & 0.090 & 0.950 & 0.691 & 0.134 & $1.32 \mathrm{E}-04$ & $2.04 \mathrm{E}-06$ & 4.60E-07 & 7.57E-01 & 2.349 \\
\hline rs330203 & 5 & 119016414 & $C / G$ & CTC-507E12.1 & 0.500 & 0.996 & -0.355 & 0.075 & $4.05 \mathrm{E}-03$ & $7.02 \mathrm{E}-06$ & $3.40 \mathrm{E}-06$ & $8.74 \mathrm{E}-01$ & 2.997 \\
\hline rs141034411 & 8 & 3098640 & $\mathrm{C} / \mathrm{A}$ & CSMD1 & 0.413 & 0.935 & 0.353 & 0.075 & 9.47E-05 & $3.20 \mathrm{E}-05$ & $3.69 \mathrm{E}-06$ & $5.71 \mathrm{E}-01$ & 2.843 \\
\hline rs2278745 & 10 & 71152091 & $\mathrm{~T} / \mathrm{C}$ & $H K 1$ & 0.602 & 0.966 & -0.395 & 0.075 & $3.05 \mathrm{E}-03$ & $5.70 \mathrm{E}-06$ & $2.95 \mathrm{E}-07$ & $1.51 \mathrm{E}-01$ & 3.668 \\
\hline rs76930569 & 15 & 28196145 & $\mathrm{C} / \mathrm{T}$ & OCA2 & 0.623 & 1.000 & 0.527 & 0.074 & $1.03 \mathrm{E}-06$ & $9.27 \mathrm{E}-11$ & $5.30 \mathrm{E}-12$ & $3.42 \mathrm{E}-01$ & 7.007 \\
\hline \multicolumn{14}{|l|}{ Delta signals } \\
\hline rs72776813 & 2 & 2792075 & $\mathrm{G} / \mathrm{A}$ & & 0.211 & 0.980 & -0.481 & 0.093 & $1.18 \mathrm{E}-03$ & $8.21 \mathrm{E}-02$ & $7.15 \mathrm{E}-02$ & $4.25 \mathrm{E}-07$ & 1.226 \\
\hline rs243946 & 4 & 111300362 & $\mathrm{C} / \mathrm{T}$ & ENPEP & 0.481 & 0.984 & -0.339 & 0.075 & $1.52 \mathrm{E}-04$ & $3.60 \mathrm{E}-03$ & 1.99E-03 & 7.74E-06 & 1.716 \\
\hline rs16904127 & 8 & 130628606 & $\mathrm{G} / \mathrm{A}$ & $C C D C 26$ & 0.469 & 0.904 & -0.359 & 0.079 & $2.30 \mathrm{E}-01$ & $8.01 \mathrm{E}-01$ & $5.78 \mathrm{E}-01$ & $7.13 E-06$ & 0.132 \\
\hline
\end{tabular}




\begin{tabular}{|c|c|c|c|c|c|c|c|c|c|c|c|c|}
\hline rs7914735 & 10 & 110441149 & $\mathrm{C} / \mathrm{T}$ & 0.537 & 0.988 & -0.356 & 0.075 & $6.46 \mathrm{E}-02$ & $3.40 \mathrm{E}-01$ & $3.54 \mathrm{E}-01$ & 3.03E-06 & -0.227 \\
\hline NA & 14 & 106875131 & $A / G$ & 0.522 & 0.884 & -0.374 & 0.081 & & & & $5.63 \mathrm{E}-06$ & \\
\hline rs11667379 & 19 & 9126468 & $\mathrm{G} / \mathrm{C}$ & 0.411 & 0.954 & 0.348 & 0.074 & $1.70 \mathrm{E}-02$ & $3.44 \mathrm{E}-01$ & $2.92 \mathrm{E}-01$ & $3.45 \mathrm{E}-06$ & 0.532 \\
\hline
\end{tabular}




\section{Table 3(on next page)}

Results of the replication effort for skin pigmentation signals in three independent Chinese samples.

Note that in the Physical Examination and Taizhou cohorts melanin was reported as M-index (higher M-values indicate darker skin), whereas in the sample of College students melanin was reported as $L^{*}$ (higher $L^{*}$ values indicate lighter skin). 
1 Table 3. Results of the replication effort for skin pigmentation signals in three independent Chinese samples. Note that in the Physical

2 Examination and Taizhou cohorts melanin was reported as M-index (higher M-values indicate darker skin), whereas in the sample of College

3 students melanin was reported as $L^{*}$ (higher $L^{*}$ values indicate lighter skin).

\begin{tabular}{|c|c|c|c|c|c|c|c|c|c|c|c|c|c|}
\hline SNP & $\mathrm{CHR}$ & POS & NEA/EA & \multirow{2}{*}{\multicolumn{2}{|c|}{$\begin{array}{l}\text { This study } \\
\text { Upper arm } \\
\end{array}$}} & \multirow{2}{*}{\multicolumn{2}{|c|}{$\begin{array}{c}\begin{array}{c}\text { Physical Examination } \\
\text { Cohort }\end{array} \\
\text { Buttocks } \\
\end{array}$}} & \multirow{2}{*}{\multicolumn{2}{|c|}{$\begin{array}{c}\text { Taizhou cohort } \\
\text { Upper arm } \\
\end{array}$}} & \multicolumn{4}{|c|}{ College Students } \\
\hline Site & & & & & & & & & & & ocks & Up & r arm \\
\hline \multirow{2}{*}{ index } & & & & \multicolumn{2}{|c|}{ M-index } & \multicolumn{2}{|c|}{ M-index } & \multicolumn{2}{|c|}{ M-index } & \multicolumn{2}{|c|}{$L^{*}$} & \multicolumn{2}{|c|}{$L^{*}$} \\
\hline & & & & Beta & $P$-value & Beta & $P$-value & Beta & $P$-value & Beta & $P$-value & Beta & $P$-value \\
\hline rs2003589 & 2 & 217527465 & $\mathrm{~T} / \mathrm{C}$ & 2.464 & $1.05 \mathrm{E}-06$ & -0.104 & 0.864 & 0.171 & 0.575 & -1.927 & 0.259 & -0.659 & 0.635 \\
\hline rs853807 & 5 & 67752638 & $\mathrm{C} / \mathrm{T}$ & -0.961 & $9.75 \mathrm{E}-06$ & -0.243 & 0.440 & -0.050 & 0.755 & 0.085 & 0.744 & -0.018 & 0.931 \\
\hline rs57836066 & 6 & 71304950 & $T / G$ & -1.838 & $2.40 \mathrm{E}-06$ & 0.605 & 0.225 & 0.213 & 0.394 & 0.995 & 0.317 & 0.175 & 0.828 \\
\hline rs2373391 & 7 & 88449300 & $\mathrm{~T} / \mathrm{A}$ & -1.076 & 5.09E-06 & -1.132 & 0.003 & 0.024 & 0.889 & 0.120 & 0.739 & 0.273 & 0.349 \\
\hline rs7945369 & 11 & 103425586 & $\mathrm{~T} / \mathrm{C}$ & -1.159 & $3.97 E-07$ & -0.614 & 0.051 & 0.211 & 0.190 & NA & NA & NA & NA \\
\hline
\end{tabular}

